\title{
The analysis of T-DNA insertional Colletotrichum gloeosporioides in Stylo pathogenicity-weakened mutant strain 1681
}

\author{
Peidong $X u^{1,2, a}$, Xiaolan Zheng ${ }^{2, b}$, Wen Tang ${ }^{2,3, c}$, Qiujie $\mathrm{Li}^{2,3, d}$, Weihuai $\mathrm{Wu}^{2, \mathrm{e}}$, \\ Jingen $\mathrm{Xi}^{2, \mathrm{f}}$, Yanqiong Liang ${ }^{2, \mathrm{~g}}$, Jinlong Zheng ${ }^{2, \mathrm{~h}}$, Rui $\mathrm{Li}^{2, \mathrm{i}}$, Chicheng Zhang ${ }^{2,3, \mathrm{j}}$, \\ Kexian $\mathrm{Yi}^{2,4, \mathrm{k}^{*}}$, Chunping $\mathrm{He}^{2, I^{*}}$, Xiaobo Zhang ${ }^{5,6, \mathrm{~m}^{*}}$ \\ ${ }^{1}$ College of Agriculture, Hainan University, Haikou, 570228, China; \\ ${ }^{2}$ Environment and Plant Protection Institute, CATAS, Haikou, 571101, China; \\ ${ }^{3}$ College of Environment and Plant Protection, Hainan University, Haikou, Hainan 570228, China \\ ${ }^{4}$ Institute of Tropical Bioscience and Biotechnology, CATAS, Haikou, 571101, China; \\ ${ }^{5}$ Key Laboratory of Protection and Development Utilization of Tropical Crop Germplasm Resources \\ (Hainan University), Ministry of Education, Haikou, 570228, China; \\ ${ }^{6}$ College of Tourism, Hainan University, Haikou, 570228, China \\ axuridongshengxpd@163.com, borchidzh@163.com, 'tangwenbendan@163.com, \\ liqiujie11@126.com, 'WeihuaiWu2002@163.com, xijingen@163.com, \\ gyanqiongliang@126.com, 'zhengjinlong_36@163.com, '809312533@qq.com, \\ jcczhang1992@126.com, kyikexian@126.com, 'hechunppp@163.com, mangiaoo@126.com \\ ${ }^{*}$ Corresponding author: Kexian Yi, Chunping He, Xiaobo Zhang \\ Peidong $\mathrm{Xu}$, Xiaolan Zheng and Wen Tang contributed equally to this work.
}

Keywords: Stylosanthes guianensias; Colletotrichum gloeosporioides; T-DNA; pathogenicity

Abstract. Biological phenotypes of mutant strain 1681 that was the pathogenicity-reduced strain in Colletotrichum gloeosporioides of Stylosanthes guianensia were analyzed by testing growth rate, colonial morphologies and sporulation ability. TAIL-PCR was used to identify the T-DNA integration site and the genes of flanking right site of the T-DNA. The sequences was analyzed by local blast and the gene and the function were predicted by bioinformatics methods. The results showed that the pathogenicity of the mutant 1681 was reduced by comparing with wild type strain $\mathrm{CH} 008$ in Colletotrichum gloeosporioides of Stylosanthes guianensis. The growth rate between strain $1681(1.42 \pm 0.02) \mathrm{cm} / \mathrm{d}$ and $\mathrm{CH} 008(1.41 \pm 0.03) \mathrm{cm} / \mathrm{d}$ had no significant difference. The sporulation between strain $1681(1.50 \pm 0.25) \times 10^{6} / \mathrm{mL}$ and $\mathrm{CH} 008(9.26 \pm 0.14) \times 10^{6} / \mathrm{mL}$ had significant difference. The spore germination rate between strain $1681(0.00 \pm 0.00) \%$ and $\mathrm{CH} 008(91.14 \pm 6.66) \%$ had significant difference. In contrast the colony diameter and colony morphology had no significant difference. Two sequences were cloned by TAIL-PCR. The length of the RB flanking sequence was $412 \mathrm{bp}$ and the LB flanking sequence was $381 \mathrm{bp}$. The blast result showed that the sequence had 100\% homology to genome of wild type strain $\mathrm{CH} 008$ in Colletotrichum gloeosporioides of Stylosanthes guianensis. The bioinformatics analysis showed the T-DNA maker the area of initial exon. It contains a complete open reading frame (ORF), encoding 682 amino acid. By predicting the function of sequence in NCBI, the blast result showed that the sequence had $99 \%$ homology to gene Nara gc5 phenylalanyl-tRNA synthetase (PheRS) beta chain core domain. It belongs to the class II aminoacyl-tRNA synthetases (aaRS) like-core super family based upon its structure.

\section{Introduction}

Stylosanthes guianensis is the high-quality leguminous forage that was widely cultivated in tropical and subtropical regions. And it was known as "The king of tropical forage" [1,2]. Stylosanthes anthracnose is a global severe disease in stylo's production and mainly caused by the pathogenic fungi Colletotrichum gloeosporioides. The pathogen can infect all parts of the stylo 
include leaves, petioles, stems and inflorescences and has a high genetic variability and biodiversity $[3,4]$. For the prevention of the anthracnose, it is difficult to have a new progress by the chemical control and the resistance breeding. So in order to understand the pathogenesis and infection of Colletotrichum gloeosporioides, more and more experts are working on the isolation and analysis of pathogencity-related gene in pathogen. ATMT, Agrobacterium tumefaciens-mediated genetic transformation, becomes an effective technique for the research to the pathogencity-related gene[5-7]. Many experts have been screened some pathogencity-related genes by ATMT[8-11], however the pathogenicity-related genes of Colletotrichum gloeosporioides in stylo has not been reported.

$\mathrm{Hu}[12]$ had a research that optimization of genetic transformation system of Stylo Anthracnose mediated by Agrobacterium tumefaciens. The screening of pathogenicity defect mutant in Colletotrichum gloeosporioides of Stylosanthes guianensias in laboratory. The pathogenicity-weakened mutant strain 1681 was selected. The molecular detection of mutant strain 1681 was identified by PCR. Biological phenotypes of 1681 were analyzed by testing colony diameter, colonial morphologies and sporulation ability. TAIL-PCR was used to identify the T-DNA integration site and the genes of flanking right site of the T-DNA. From that, we hope to provide a theoretical basis for the research of the cloning and functional analyse of pathogenicity-related genes of Colletotrichum gloeosporioide. And we hope to provide some molecular evidence to understand the pathogenesis and infection of Colletotrichum gloeosporioides.

\section{Materials and methods}

\section{Materials}

Strains The strains includes wild type strain CH008 and mutant strain 1681. The wild type strain CH008 is the aggressively virulent strain of Colletotrichum gloeosporioides[13]. An Agrobacterium tumefaciens AGL-1 which contain binary vector with ILV1 and GFP as report gene was used for transformation of Colletotrichum gloeosporioides[14]. All Strains were stored at $-70^{\circ} \mathrm{C}$ lab freezer.

Mediums Luria-Bertani Medium(LB): Peptone $10.0 \mathrm{~g}$, Yeast extract $5.0 \mathrm{~g}$, NaCl $8.0 \mathrm{~g}$, Agar 20 g, Add ddH $\mathrm{H}_{2} \mathrm{O}$ to $1000 \mathrm{~mL}, \mathrm{pH} 7.0$. Potato dextrose agar( PDA): Potato $200 \mathrm{~g}$, Glucose $15 \mathrm{~g}$, Agar powder $20 \mathrm{~g}$, Add $\mathrm{ddH}_{2} \mathrm{O}$ to $1000 \mathrm{~mL}$.

\section{Primers and Main reagents}

All primers used in the research can be found in table 1. The primer synthesis and sequencing were done by Life Technologies Trading (Shanghai) Corporation. DNA Maker and Taq DNA polymerase were obtained from TaKaRa. Fungle gDNA Kit were bought from Biomiga Corporation. Plasmid Mini Kit I were bought from Omega Corporation Corporation. The pEASY-T1 Cloning vector and E. coli trans T1 were bought from TransGen Biotech (Beijing) Corporation. 
Table 1 Primers used in the research

\begin{tabular}{ll}
\hline Primer name & Sequence $\left(5^{\prime} \rightarrow 3^{\prime}\right)$ \\
\hline GFP $_{1}$ & TACTGCAGATGGTGAGCAAGGGCGAG \\
GFP $_{2}$ & CGGGATCCCTTGTACAGCTCGTCCATG \\
LB1 & GGGTTCCTATAGGGTTTCGCTCATG \\
LB2 & CATGTGTTGAGCATATAAGAAACCCT \\
LB3 & GAATTAATTCGGCGTTAATTCAGT \\
RB1 & GGCACTGGCCGTCGTTTTACAAC \\
RB2 & AACGTCGTGATGGGAAAACCCT \\
RB3 & CCCTTCCCAACAGTTGCGCA \\
AD1 & AGWGNAGWANCAWAGG \\
AD2 & WAGTGNAGWANCANAGA \\
AD3 & WAGTGNAGWANCANGTT \\
AD4 & TGWGNAGWANCASAGA \\
AD8 & STTGNTASTNCTNTGC \\
AD9 & WCAGNTGWTNGTNCTG \\
M13F & ACTGGCCGTCGTTTTAC \\
M13R & GTCATAGCTGTTTCCTG \\
\hline
\end{tabular}

Note: $\mathrm{W}=\mathrm{A}$ or $\mathrm{T} ; \mathrm{S}=\mathrm{G}$ or $\mathrm{C} ; \mathrm{N}=\mathrm{A}, \mathrm{T}, \mathrm{G}$ or $\mathrm{C}$

\section{Methods}

The pathogenicity analysis of mutant strain The pathogenicity analysis of mutant strain was determined by inoculating pathogen on detached leaves. The variety of Stylosanthes guianensia is Gram. The ternate pinnate leaves of Stylo with the leaf age of 7 days were selected. Mycelium cakes at the edge of bacterial colony were cultivated on PDA for 5 days. The surface of a mycelium cake, which contained hyphae, was attached to leaves with punctured parts and normal ones to observe morbidity. This experiment was performed three replicates.

The molecular detection of mutant strain For DNA extraction, fungal isolates were grown in PDA liquid medium for 4 days at $28^{\circ} \mathrm{C}$ in $180 \mathrm{r} / \mathrm{min}$. DNA was extracted from freezedried mycelium using the Fungle gDNA Kit, according to the manufacturer's instructions.PCR primers $\mathrm{GFP}_{1}$ and $\mathrm{GFP}_{2}$ were designed from the T-DNA which containing binary vector with ILV1 and GFP as report gene. Each PCR $(20 \mu \mathrm{L})$ contained $10 \times \operatorname{Buffer}(2.0 \mu \mathrm{L})$, dNTPs $(1.2 \mu \mathrm{L}), 0.5 \mu \mathrm{L}$ each primer $(20 \mu \mathrm{mol} / \mu \mathrm{L})$, $0.2 \mu \mathrm{L}$ Taq DNA polymerase, $1 \mu \mathrm{L}$ DNA, Add ddH2O to $20 \mu \mathrm{L}$. The DNA was amplified with 35 cycles using the following conditions: Amplified procedure was programmed for $4 \mathrm{~min}$ at $94^{\circ} \mathrm{C}$; denaturation at $94^{\circ} \mathrm{C}$ for $30 \mathrm{~s}$, annealing at $58^{\circ} \mathrm{C}$ for $30 \mathrm{~s}$, elongation at $72^{\circ} \mathrm{C}$ for $45 \mathrm{~s}$ and a final elongation step at $72^{\circ} \mathrm{C}$ for $5 \mathrm{~min}$. PCR products were visualized by electrophoresis on $1.0 \%$ (wt/vol) agarose gels.

\section{The phenotypes of pathogenicity- defective mutant strain}

The wild type strain $\mathrm{CH} 008$ and the mutant strain 1681 were cultivated on PDA at $28^{\circ} \mathrm{C}$ for 6 days. Mycelium cakes(diameter, $0.5 \mathrm{~cm}$ ) at the edge of strain were taken. All experiments were performed three replicates. Data were analyzed using analysis of variance and the Tukey honest significant difference test with DPS(7.05).

Assessment of morphological and cultural characteristics. The colony diameter (three replicates; four measurements per replicate with statistical analysis as above) and characteristics (texture, color, presence of conidial masses and color of the reverse side) were recorded from cultures grown for 6 days at $28^{\circ} \mathrm{C}$ under darkness on PDA plates $(9 \mathrm{~cm})$.

Conidial phenotypes assays. Conidia were collected in $5 \mathrm{~mL}$ of distilled water by scraping PDA. And conidia were counted with a haemacytometer under a microscope.

Spore germination of pathogenic mutants. Conidia harvested from PDB medium were diluted into $1.0 \times 10^{5}$ conidia per milliliter in sterile distilled water. Drops of conidial suspension $(30 \mu \mathrm{L})$ were placed on the coverslips with three replicates, then placed in a moistened box and incubated at $28^{\circ} \mathrm{C}$. After 8 hours incubation, the percentage of conidial germination and appressorium formation was 
determined by microscopic examination of at least 100 conidia per replicate in at least three independent experiments.

Amplification of flanking sequences by TAIL-PCR. Cloning of flanking sequences at T-DNA insertion sites of mutant strains with pathogenic defects. A thermal asymmetric interlaced PCR (TAIL-PCR) protocol was used for amplification of the flanking sequences at T-DNA insertion sites. The right border primers (RB1, RB2 and RB3) and the left border primers (LB1, LB2 and LB3) were used as methods of Mullins[15] and Combier [16]. Six arbitrary degenerate primers were designed such that their melting temperatures would ensure maximum thermal asymmetric priming and ensure cloning and sequencing of genomic DNA flanking T-DNA. PCR products were visualized by electrophoresis on $1.0 \%$ (wt/vol) agarose gels. And then cloning and sequencing were conducted. BLAST comparison was implemented for the obtained sequence.

\section{Results}

The pathogenicity analysis of mutant strain 1681. The pathogenicity analysis of mutant strain was determined by inoculating pathogen on detached leaves. The result of the pathogenicity analysis of mutant strain 1681 is shown in Fig.1. There are clear brown spots on the ternate pinnate leaves of Stylo at the infected part of wild strain $\mathrm{CH} 008$. And spots range from the blade extends to petiole. The area of the brown spots was significantly reduced at the infected part of mutant strain 1681 . Three replicates later, we got the same result. In brief, comparing with wild type strain $\mathrm{CH} 008$ in Colletotrichum gloeosporioides of Stylosanthes guianensis, the pathogenicity of the mutant 1681 was reduced.

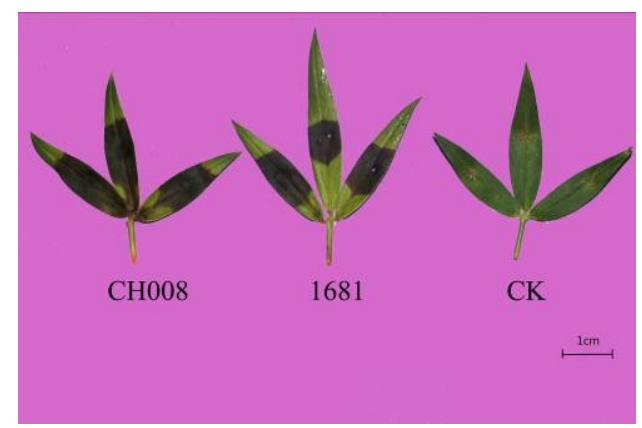

Fig.1 The pathogenicity analysis of mutant strain 1681

The molecular detection of mutant strain 1681. The DNA of the wild type strain CH008 and the mutant strain 1681 were extracted from freezedried mycelium using the Fungle gDNA Kit. The result of PCR showed that a gene fragment of the 1861 with molecular size about 750 bp ( Fig.2) were obtained after PCR amplifications. But the wild type strain $\mathrm{CH} 008$ did not demonstrate bright and clear strips at $750 \mathrm{bp}$. So the T-DNA stably inserted into the genome of 1681 .

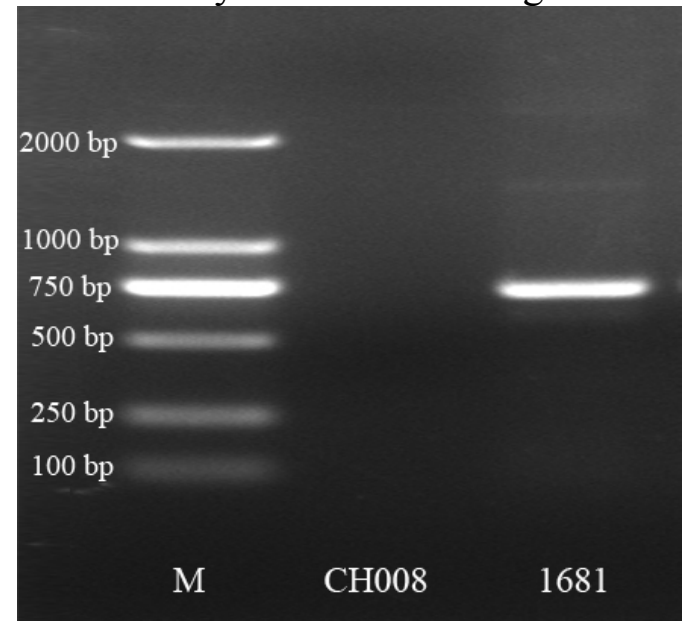

Fig.2 The molecular detection of mutant strain 1681 
The phenotypes of pathogenicity- defective mutant strain 1681. Biological phenotypes of mutant strain 1681 were analyzed by testing colony diameter, colony morphology, conidia production and spore germination. Data were analyzed by using analysis of variance and the Tukey honest significant difference test with DPS(7.05). The results showed that by comparing with wild type strain CH008, the growth rate between strain $1681(1.42 \pm 0.02) \mathrm{cm} / \mathrm{d}$ and $\mathrm{CH} 008(1.41 \pm 0.03) \mathrm{cm} / \mathrm{d}$ had no significant difference(Fig.3A,Table 2). The mycelium of $\mathrm{CH} 008$ was dense and a large number of spores produced could be observed under the mycelium. However there are not spores of 1681 could be observed under the mycelium. And the colonial morphologies had no significant difference.

Conidia were collected in $5 \mathrm{~mL}$ of distilled water by scraping PDA. And conidia were counted with a haemacytometer under a microscope. The results showed that the sporulation between strain $1681(1.50 \pm 0.25) \times 10^{6} / \mathrm{mL}$ and $\mathrm{CH} 008(9.26 \pm 0.14) \times 10^{6} / \mathrm{mL}$ had significant difference(Fig.3B, Table 2). These results suggested that the site of T-DNA insertion mutant 1681 may lead to reduced sporulation.

Drops of conidial suspension were placed on the coverslips with three replicates, then placed in a moistened box and incubated at $28^{\circ} \mathrm{C}$. After 8 hours incubation, the results showed that the spore germination rate between strain $1681(0.00 \pm 0.00) \%$ and $\mathrm{CH} 008(91.14 \pm 6.66) \%$ had significant difference(Fig.3C, Table 2). However after 24 hours incubation, there are not appressorium formination of strain $\mathrm{CH} 008$ and 1681. Relatively speaking, these results suggested that the site of T-DNA insertion mutants 1681 may lead to reduced the spore germination rate.

A
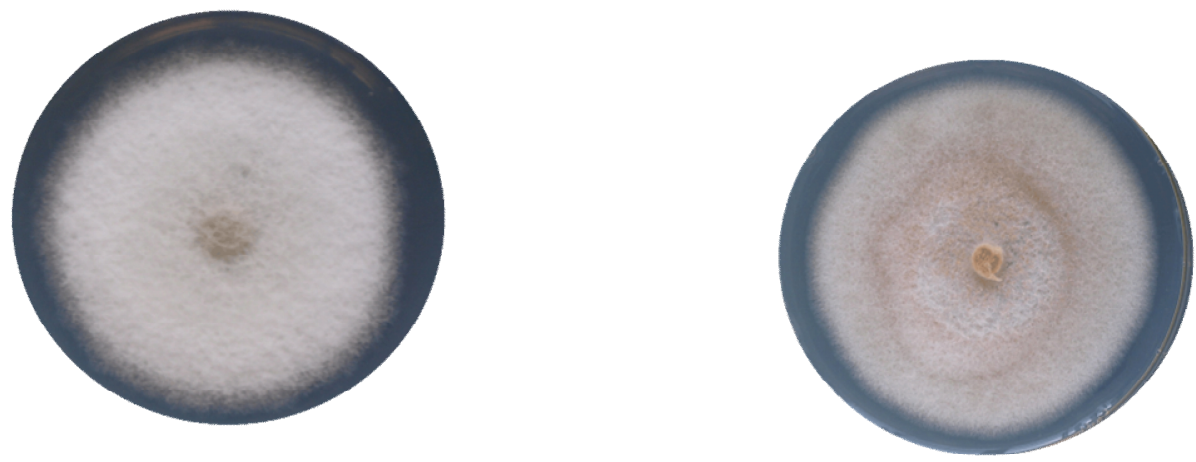

$\mathrm{B}$
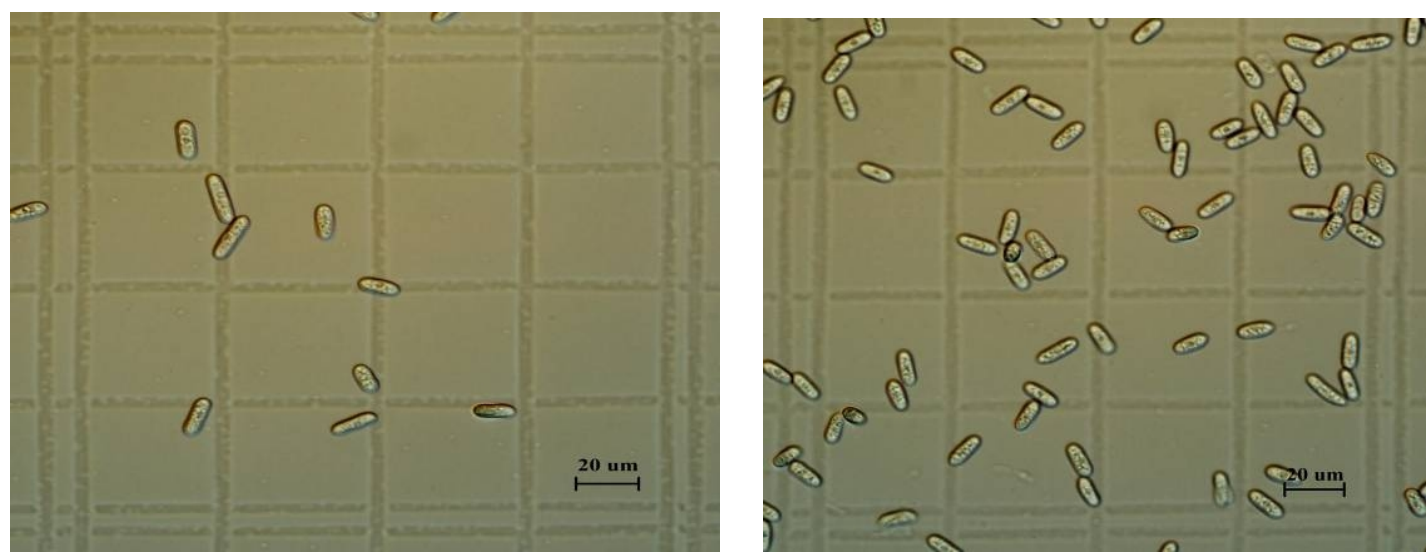

$\mathrm{C}$ 


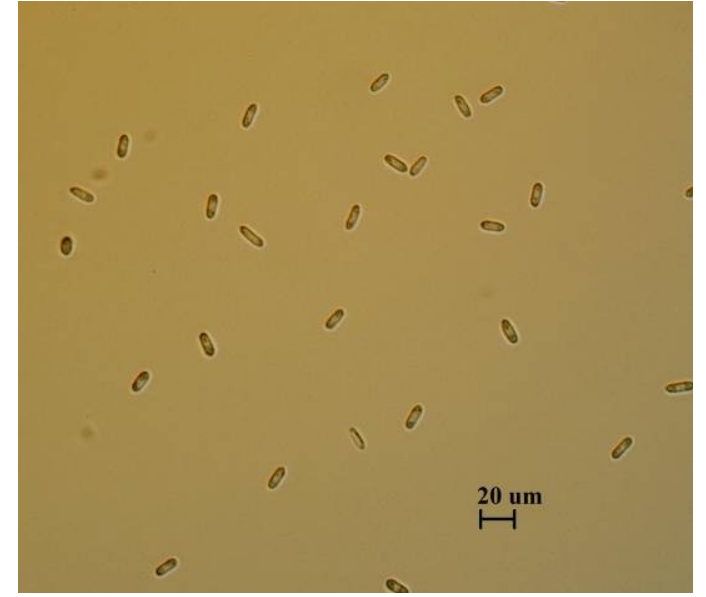

1681

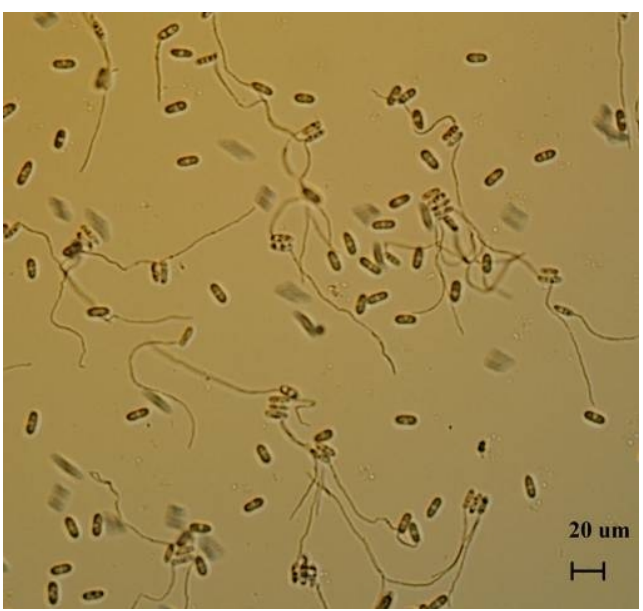

$\mathrm{CH} 008$

A: Phenotypic analysis diameters on PDA medium; B: Statistical analysis of conidia production; C:

Spore germination of pathogenic mutants

Fig.3 The phenotypes of pathogenicity- defective mutant strain 1681

Table 2 The significant difference of phenotypes between 1681 to $\mathrm{CH008}$

\begin{tabular}{cccc}
\hline Strain & Growth rate $(\mathrm{cm} / \mathrm{d})$ & Sporulation $\left(\times 10^{6}\right)$ & Spore germination $(\%)$ \\
\hline CH008 & $1.41 \pm 0.03 \mathrm{aA}$ & $9.26 \pm 0.14 \mathrm{aA}$ & $91.14 \pm 6.66 \mathrm{aA}$ \\
1681 & $1.42 \pm 0.02 \mathrm{aA}$ & $1.50 \pm 0.25 \mathrm{bB}$ & $0.00 \pm 0.00 \mathrm{bB}$ \\
\hline
\end{tabular}

Note: Small letters represent the statistic difference at 0.05 level; capital letters indicate the statistic difference at 0.01 level.

Cloning of flanking sequences at T-DNA insertion sites of mutant strain 1681. TAIL-PCR was used for amplification of the flanking sequences at T-DNA insertion sites of mutant strain 1681. The effective random primer that the right flanking sequence was amplified was AD4 and another effective random primer by which the left flanking sequence was amplified was AD9. And the right flanking sequences of $600 \mathrm{bp}$ and the left flanking sequences of $500 \mathrm{bp}$ were obtained(Fig.4).
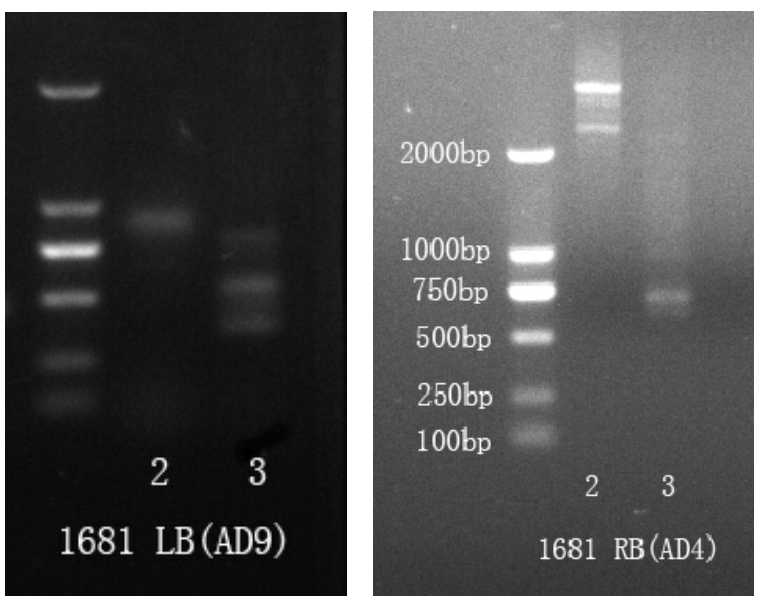

Fig.4 The TAIL-PCR amplification results of mutant strain 1681

Note: M: Maker; L-2/ R-2: The LB or RB flanking sequence of NO.2; L-3/R-3: The LB or RB flanking sequence of NO.3.

The prediction and analysis of inserted site gene. The PCR products were cloned into T-vector and sequenced. The sequencing results showed that the right flanking sequence was $412 \mathrm{bp}$ and the left flanking sequence was $381 \mathrm{bp}$. Two individual sequence fragments were assembled into entire sequence. The blast result showed that the sequence had $100 \%$ homology to genome of wild type strain CH008 in Colletotrichum gloeosporioides of Stylosanthes guianensis. The website 'The FGENESH Program' (Softberry Inc., Mount Kisco,NY, USA; http://linux1.Softberry.com/ berry.phtml) was used to predict its inserted site gene. We get 2505 bp gene sequence with the 
Magnaporthe as a model to predict. The bioinformatics analysis showed the T-DNA maker is in the area of initial exon. It contains a complete open reading frame (ORF), encoding 682 amino acid. By predicting the function of sequence in NCBI, the blast result showed that the sequence had $99 \%$ homology to gene Nara gc5 phenylalanyl-tRNA synthetase (PheRS) beta chain core domain. It was called $S t C g$ - $X 1681$ and belongs to the class II aminoacyl-tRNA synthetases (aaRS) like-core super family based upon its structure.

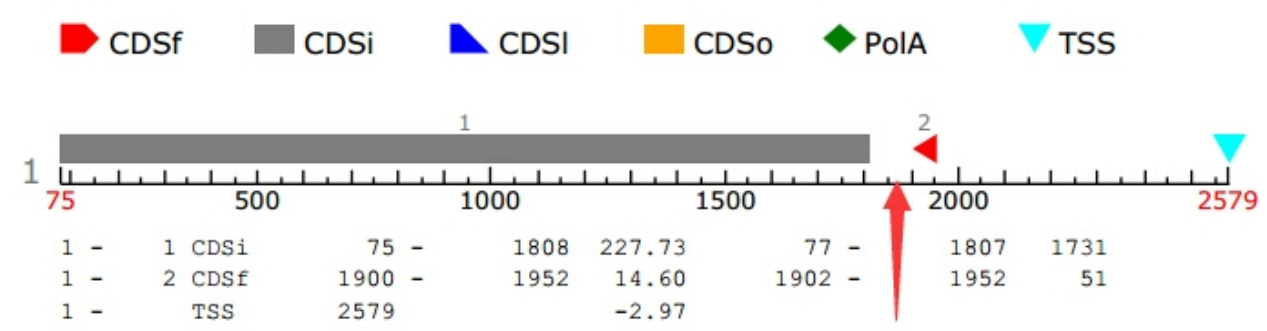

Fig.5 The prediction and analysis of the T-DNA inserted site gene of mutant strain 1681

\section{Conclusions}

The plants are infected by the fungus in many different ways. In the penetration period, spores attach to the surface of host and germinate the germ tube which eventually turn to different appressoria[17]. The penetration peg between appressoria and plants will penetrate the cuticle and then become into intracellular hemibiotrophic pathogens or subcutitle intramural pathogens[18]. That means the change of Biological traits will lead to the different infections by the Colletotrichum gloeosporioides and the change of pathogenicity-defective mutant strain will have different biological traits in the other way[19]. In this research, by comparing with wild type strain CH008 in Colletotrichum gloeosporioides of Stylosanthes guianensis, the pathogenicity of the mutant 1681 was reduced. The growth rate and the colonial morphologies had no significant difference. In comparison, the sporulation and the spore germination had significant difference.

Currently the study in China and other countries have made great progress about analyze the genovariation of mutant strain though ATMT. The anthrax pathogenicity-related genes $C g D N 3[8]$, CPRI[9],CPR1[10], PKSI[11], etc has been found though T-DNA inserted site gene while a pathogenicity-related genes of Stylo have not been found any way. In this study, the PCR test of the mutant strain 1681 shows that the T-DNA has already inserted. TAIL-PCR is widely used in the amplification and cloning of unknown flanking sequences and the optimum random primer is the key to success[20]. In this research, two sequences were cloned by TAIL-PCR. The effective random primer that the right flanking sequence was amplified was AD4 and another effective random primer that the left flanking sequence was amplified was AD9. The length of the RB flanking sequence was $412 \mathrm{bp}$ and the LB flanking sequence was $381 \mathrm{bp}$.

In this research, the blast result showed that the sequence had 100\% homology to genome of wild type strain CH008 in Colletotrichum gloeosporioides of Stylosanthes guianensis. We got 2505 bp gene sequence with the Magnaporthe as a model to predict. The bioinformatics analysis showed the T-DNA maker is in the area of initial exon. It contains a complete open reading frame (ORF), encoding 682 amino acid. By predicting the function of sequence in NCBI, the blast result showed that the sequence had $99 \%$ homology to gene Nara gc5 phenylalanyl-tRNA synthetase (PheRS) beta chain core domain. It was called $S t C g$-X1681 and belongs to the class II aminoacyl-tRNA synthetases (aaRS) like-core super family based upon its structure.

\section{Acknowledgements}

This research was supported by the National Natural Science Foundation of China (31072076, 31101408), Special Fund for Agro-scientific Research in the Public Interest (201303057) and the basic scientific research funded projects (2015hzs1J002). 


\section{References}

[1] F M Liu, X D Cui, K R Wu, et al. Biological characteristics of Colletotrichum gloeosporioides Penz. causing anthracnose of Stylosanthes guianensis. Guangdong Agricultural Science, 2012, 16: 65-68.

[2] K X Yi. Development and prospects and countermeasures on tropical forage industrial production in China. Chinese Journal of Tropical Agricultur, 2001,4: 30-34.

[3] C S Jiang, D M Zhou, Z Y Zhang. Stylo anthracnose and the progress of its breeding for resistance to disease. Journal of Sichuan Grassland,2002, (1):22-27.

[4] Chakraborty S, Perrott R, Charchar M J D, et al. Biodiversity, epidemiology and virulence of Colletotrichum gloeosporioides. II.Genetic and pathogenic diversity in isolates of Colletotrichum gloeosporioides from eight species of stylosanthes.Tropical Grasslands, 1997, 31: 393-401.

[5] X L Chen, J Yang, Y L Peng. Large-scale insertional mutagenesis in Magnaporthe oryzae by Agrobacterium tumefaciens-mediated transformation. Methods in Molecular Biology, 2011, 722: 213-224.

[6] P J Liu, Z Y Wang, Q H Wang, et al. Agrobacterium tumefaciens mediated transformation of Magnaporthe grisea and identification of pathogenicity defective mutant. Chinese Journal of Rice Science, 2006, 20(3): 231-237.

[7] M N Yu, J K Hu, L Huang, et al. Molecular characterization of T-DNA integration of the Ustilaginoidea virens Mutant 5062. Scientia Agricultura Sinica, 2013, 46(9): 1790-1798.

[8] Stephenson S A, Hatfield J, Rusu A G, et al.CgDN3: An essential pathogenieity gene of Colletotrichum gloeosporioides neeessary to avert a hypersensitive-like response in the host Stylosanthes guianensis. Molecular Plant-Microbe Interactions, 2000, 13: 929-941.

[9] Thon M R, Nuekles E M, Takaeh J E, et al. CPR I:A gene encoding a putative signal peptidase that functions in pathogenicity of Colletotrichum graminicola to maize. Molecular Plant-Microbe Interactions, 2002, 15: 120-128.

[10] Thon M R, Nuckles E M, Takach J E, et al. CPRl: A gene encoding a putative signal peptidase that functions in pathogenicity of Colletotrichum graminicola to maize. Molecular Plant-Microbe Interactions, 2002, 15: 120-128.

[11] Tsuji G, Fujii S, Tsuge S, et al. The Colletotrichum lagenarium Ste12-Like gene CSTl is essential for appressorium penetration. Molecular Plant-Microbe Interactions, 2003, 16: 315-325.

[12] C P Hu, J L Zheng, J M Gao, et al. Optimization of genetic transformation system of Stylo Anthracnose mediated by Agrobacterium tumefaciens.Chinese Journal of Tropical Crops, 2013, 34(6): 1007-1012.

[13] K X Yi, J S Huang, G D Liu. Genetic diversity analysis of Chinese Stylo anthracnose pathogens using random amplified polymorphic DNA.Acta Microbiologica Sinica, 2003,43(3):379-387.

[14] J M Gao, C P Hu, J L Zheng, et al. Construction and analysis of a T-DNA insertional mutant library for Colletotrichum gloeosporioides. Guangdong Agricultural Science, 2014, 19(10): 142-145.

[15] Mullins E D, Chen P X, Romaine P, et al. Agrobacterium-mediated transformation of Fusarium oxysporum: An efficient tool for inserlional mutagenesis and gene transfer. Phytopathology, 2001, 91: 173-180.

[16] Combier J P, Melayah D, Raffier C, et al. Agrobacterium tumefaciens -mediated transformation as a tool for insertional mutagenesis in the symbiotic ectomycorrhizal fungus Hebeloma Cylindrosporum.FEMS Microbiology Letters, 2003, 220(1): 141-148.

[17] M Liu, W Zhang, Y Zhou, et al. Research progress on grape anthracnose. China Plant Protection, 2014,34(1): 29-33.

[18] Wharton P S, Julian A M. A cytological study of compatible and incompatible interactions between Sorghum bicolor and Colletotrichum sublineolum. New Phytologist, 1996,134(1): 25-34. 
[19] Kim Y K, Wang Y H, Liu Z M, et al. Identification of a hard surface contact-induced gene in Colletotrichum gloeosporioides conidia as a sterol glycosyl transferase, a novel fungal virulence factor. The Plant Journal, 2002, 30: 177-187.

[20] B Y Sun, H L Piao, Park S H, et al. Selection of optimal primers for TAIL-PCR in identifying Ds flanking sequences from Ac/Ds insertion rice lines.Chinese Journal of Biotechnology, 2004, 20(6): 821-826. 